\title{
On the quenched CLT for stationary random fields under projective criteria
}

\author{
Na Zhang*, Lucas Reding, and Magda Peligrad \\ Department of Mathematics, Towson University, Towson, MD 21252-0001,USA. \\ Email: nzhang@towson.edu* \\ Université de Rouen Normandie. F76801 Saint-Étienne-du-Rouvray. \\ Email: lucas.reding@etu.univ-rouen.fr \\ University of Cincinnati, PO box 210025, Cincinnati, OH 45221-0025, USA. \\ Email: peligrm@ucmail.uc.edu
}

\begin{abstract}
Motivated by random evolutions which do not start from equilibrium, in a recent work, Peligrad and Volný (2018) showed that the central limit theorem (CLT) holds for stationary ortho-martingale random fields when they are started from a fixed past trajectory. In this paper, we study this type of behavior, also known under the name of quenched CLT, for a class of random fields larger than the ortho-martingales. We impose sufficient conditions in terms of projective criteria under which the partial sums of a stationary random field admit an ortho-martingale approximation. More precisely, the sufficient conditions are of the Hannan's projective type. We also discuss some aspects of the functional form of the quenched CLT. As applications, we establish new quenched CLT's and their functional form for linear and nonlinear random fields with independent innovations.
\end{abstract}

Key words: random fields, quenched central limit theorem, ortho-martingale approximation, projective criteria.

Mathematical Subject Classification(2000): 60G60, 60F05, 60G42, 60G48, 41A30.

\section{Introduction}

An interesting problem, with many practical applications, is to study limit theorems for processes conditioned to start from a fixed past trajectory. This problem is difficult, since the stationary processes started from a fixed past trajectory, or from a point, are no longer stationary. Furthermore, the validity of a limit theorem is not enough to assure that the convergence still holds when the process is not started from its equilibrium. This type of convergence is also known under the name of almost sure conditional limit theorem or the quenched limit theorem. The issue of the quenched CLT for stationary processes has been widely explored for the last few decades. Among many others, we mention papers by Derriennic and Lin (2001), Cuny and Peligrad (2012), Cuny and Volný (2013), Cuny and Merlevède (2014), Volný and Woodroofe (2014), Barrera et al. (2016). Some of these results were surveyed in Peligrad (2015).

A random field consists of multi-indexed random variables $\left(X_{\mathbf{u}}\right)_{\mathbf{u} \in Z^{d}}$, where $d$ is a positive integer. The main difficulty when analyzing the asymptotic properties of random fields, is the fact that the future and the past do not have a unique interpretation. To compensate for the lack of ordering of the filtration, it is customary to use the notion of commuting filtrations. Traditionally, this

\footnotetext{
${ }^{*}$ Corresponding author
} 
kind of filtration is constructed based on random fields which are functions of independent and identically distributed random variables. Alternatively, commuting filtrations can be induced by stationary random fields with independent columns or rows. See for example, El Machkouri et al. (2013) and Peligrad and Zhang (2018a). As in the case of random processes, a fruitful approach for proving limit theorems for random fields is via the martingale approximation method, which was started by Rosenblatt (1972) and its development is still in progress. Recently, the interest is in the approximation by ortho-martingales which were introduced by Cairoli (1969). We would like to mention several important recent contributions in this direction by Gordin (2009), Volný and Wang (2014), Volný (2015), Cuny et al. (2015), Peligrad and Zhang (2018a), Giraudo (2017) and Peligrad and Zhang (2018b). However, the corresponding quenched version of these results have rarely been explored. To the best of our knowledge, so far, the only quenched invariance principle for random fields is due to Peligrad and Volný (2018). Their paper contains a quenched functional CLT for ortho-martingales and a quenched functional CLT for random fields via co-boundary decomposition. By constructing an example of an ortho-martingale which satisfies the CLT but not its quenched form, Peligrad and Volný (2018) showed that, contrary with the one dimensional index set, the finite second moment condition is not enough for the quenched CLT. For the validity of this type of results, they provided a minimal moment condition, that is: $E\left(X_{\mathbf{0}}^{2} \log ^{d-1}\left(1+\left|X_{\mathbf{0}}\right|\right)\right)<\infty$, where $\mathbf{0}=(0, \cdots, 0) \in Z^{d}$ and $d$ is the dimension.

Here, we aim to establish sufficient conditions in terms of projective criteria such that a quenched CLT holds. One of the results of this paper is a natural extension of the quenched CLT for orthomartingales in Peligrad and Volný (2018) to more general random fields under the generalized Hannan projective condition (1973). Our result is also a quenched version of the main theorem in Peligrad and Zhang (2018a). The functional form of a quenched CLT that we shall use in our applications will also be explored in this paper. The tools for proving these results consist of orthomartingale approximations, projective decompositions and ergodic theorems for Dunford-Schwartz operators.

Our paper is organized as follows. In the next section, we introduce the preliminaries and our main results for double-indexed random fields. In Section 3, we prove the quench CLT's for doubleindexed random fields. Extensions to general indexed random fields and their proofs are given in Section 4. Section 5 contains a functional CLT which will be used in applications. In Section 6, we apply our results to linear and Volterra random fields with independent innovations, which are often encountered in economics. For the convenience of the reader, in the Appendix, we provide a well-known inequality for martingales and an important theorem in decoupling theory which will be of great importance for the proof of our main results.

\section{Preliminaries and Results}

For the sake of clarity, especially due to the complicated notation, in this section, we shall only talk about the double-indexed random fields. After obtaining results for double-indexed random fields, we will extend them to random fields indexed by $Z^{d}, d>2$. We shall introduce first a stationary random field adapted to a stationary filtration. In order to construct a flexible filtration it is customary to start with a stationary real valued random field $\left(\xi_{n, m}\right)_{n, m \in Z}$ defined on a probability space $(\Omega, \mathcal{K}, P)$ and define the filtrations

$$
\mathcal{F}_{k, \ell}=\sigma\left(\xi_{j, u}: j \leq k, u \leq \ell\right) .
$$

For all $i, j \in Z$, we also define the following sigma algebras generated by the union of sigma algebras: $\mathcal{F}_{\infty, j}=\vee_{n \in Z} \mathcal{F}_{n, j}, \mathcal{F}_{i, \infty}=\vee_{m \in Z} \mathcal{F}_{i, m}$ and $\mathcal{F}_{\infty, \infty}=\vee_{i, j \in Z} \mathcal{F}_{i, j}$. 
To ease the notation, sometimes the conditional expectation will be denoted by

$$
E_{a, b} X=E\left(X \mid \mathcal{F}_{a, b}\right) .
$$

In addition we consider that the filtration is commuting in the sense that

$$
E_{u, v} E_{a, b} X=E_{a \wedge u, b \wedge v} X
$$

where the symbol $a \wedge b$ stands for the minimum between $a$ and $b$. As we mentioned before, this type of filtration is induced, for instance, by an initial random field $\left(\xi_{n, m}\right)_{n, m \in Z}$ of independent random variables or more generally can be induced by stationary random fields $\left(\xi_{n, m}\right)_{n, m \in Z}$ where only the columns are independent, i.e. $\bar{\eta}_{m}=\left(\xi_{n, m}\right)_{n \in Z}$ are independent. This model often appears in statistical applications when one deals with repeated realizations of a stationary sequence.

It is interesting to point out that commuting filtrations can be described by the equivalent formulation: for $a \geq u$ we have

$$
E_{u, v} E_{a, b} X=E_{u, b \wedge v} X .
$$

This follows from the Markovian-type property (see for instance Problem 34.11 in Billingsley, 1995).

Without restricting the generality we shall define $\left(\xi_{\mathbf{u}}\right)_{\mathbf{u} \in Z^{2}}$ in a canonical way on the probability space $\Omega=R^{Z^{2}}$, endowed with the $\sigma$-field, $\mathcal{B}(\Omega)$, generated by cylinders. Now on $R^{Z^{2}}$ we shall introduce the operators

$$
T^{\mathbf{u}}\left(\left(x_{\mathbf{v}}\right)_{\mathbf{v} \in Z^{2}}\right)=\left(x_{\mathbf{v}+\mathbf{u}}\right)_{\mathbf{v} \in Z^{2}} .
$$

Two of them will play an important role in our paper namely, when $\mathbf{u}=(1,0)$ and when $\mathbf{u}=(0,1)$. By interpreting the indexes as notations for the lines and columns of a matrix, we shall call

$$
T\left(\left(x_{u, v}\right)_{(u, v) \in Z^{2}}\right)=\left(x_{u+1, v}\right)_{(u, v) \in Z^{2}}
$$

the vertical shift and

$$
S\left(\left(x_{u, v}\right)_{(u, v) \in Z^{2}}\right)=\left(x_{u, v+1}\right)_{(u, v) \in Z^{2}}
$$

the horizontal shift.

Now we introduce the stationary random field $\left(X_{\mathbf{m}}\right)_{\mathbf{m} \in Z^{2}}$ in the following way. For a real-valued measurable function $f$ on $R^{N^{2}}$, we define

$$
X_{j, k}=f\left(T^{j} S^{k}\left(\xi_{a, b}\right)_{a \leq 0, b \leq 0}\right) .
$$

The variable $X_{0,0}$ will be assumed to be square integrable (in $L^{2}$ ) and with mean 0 . We notice that the variables $\left(X_{n, m}\right)_{n, m \in Z}$ are adapted to the filtration $\left(\mathcal{F}_{n, m}\right)_{n, m \in Z}$.

Let $\phi:[0, \infty) \rightarrow[0, \infty)$ be a Young function, that is, a convex function satisfying

$$
\lim _{x \rightarrow 0} \frac{\phi(x)}{x}=0 \text { and } \lim _{x \rightarrow \infty} \frac{\phi(x)}{x}=\infty .
$$

We shall define the Luxemburg norm associated with $\phi$ which will be needed in the sequel. For any measurable function $f$ from $\Omega$ to $R$, the Luxemburg norm of $f$ is defined by (see relation 9.18 and 9.19 on page 79 of Krasnosel'skii and Rutitskii (1961))

$$
\|f\|_{\phi}=\inf \{k \in(0, \infty): E \phi(|f| / k) \leq 1\} .
$$

In the sequel, we use the notations

$$
S_{k, j}=\sum_{u, v=1}^{k, j} X_{u, v}, P^{\omega}(\cdot)=P\left(\cdot \mid \mathcal{F}_{0,0}\right)(\omega) \text { for any } \omega \in \Omega .
$$


Also, we shall denote by $E^{\omega}$ the expectation corresponding to $P^{\omega}$ and $\Rightarrow$ the convergence in distribution.

For an integrable random variable $X$, we introduce the projection operators defined by

$$
\begin{aligned}
P_{\tilde{0}, 0}(X) & :=\left(E_{0,0}-E_{-1,0}\right)(X) \\
P_{0, \tilde{0}}(X) & :=\left(E_{0,0}-E_{0,-1}\right)(X) .
\end{aligned}
$$

Note that, by (2), we have

$$
\mathcal{P}_{\mathbf{0}}(X):=P_{\tilde{0}, 0} \circ P_{0, \tilde{0}}(X)=P_{0, \tilde{0}} \circ P_{\tilde{0}, 0}(X)=\left(E_{0,0}-E_{0,-1}-E_{-1,0}+E_{-1,-1}\right)(X) .
$$

Then for $(u, v) \in Z^{2}$, we can define the projections $\mathcal{P}_{u, v}$ as follows

$$
\mathcal{P}_{u, v}(\cdot):=\left(E_{u, v}-E_{u, v-1}-E_{u-1, v}+E_{u-1, v-1}\right)(\cdot)
$$

We shall introduce the definition of an ortho-martingale, which will be referred to as a martingale with multiple indexes or simply martingale.

Definition 2.1 Let d be a function and define

$$
D_{n, m}=d\left(\xi_{i, j}, i \leq n, j \leq m\right) .
$$

Assume integrability. We say that $\left(D_{n, m}\right)_{n, m \in Z}$ is a field of martingale differences if $E_{a, b}\left(D_{n, m}\right)=0$ if either $a<n$ or $b<m$.

Set

$$
M_{k, j}=\sum_{u, v=1}^{k, j} D_{u, v}
$$

Definition 2.2 We say that a random field $\left(X_{n, m}\right)_{n, m \in Z}$ defined by (3) admits a martingale approximation if there is a field of martingale differences $\left(D_{n, m}\right)_{n, m \in Z}$ defined by (5) such that

$$
\lim _{n \wedge m \rightarrow \infty} \frac{1}{n m} E^{\omega}\left(S_{n, m}-M_{n, m}\right)^{2}=0 \text { for almost all } \omega \in \Omega .
$$

The following theorem is an extension of the quenched CLT for ortho-martingales in Peligrad and Volný (2018) to stationary random fields satisfying the generalized Hannan condition (1973). It also can be viewed as a random field version of Proposition 11 in Cuny and Peligrad (2012) (see also Volný and Woodroofe (2014)).

Throughout the paper we shall assume the setting above namely:

Condition A. $\left(X_{n, m}\right)_{n, m \in Z}$ is defined by (3), the filtrations are commuting and either $T$ or $S$ is ergodic.

Theorem 2.3 Assume Condition $A$ and in addition

$$
\sum_{u, v \geq 0}\left\|\mathcal{P}_{0,0}\left(X_{u, v}\right)\right\|_{2}<\infty
$$

Then, for almost all $\omega \in \Omega$,

$$
\frac{1}{n} \bar{S}_{n, n} \Rightarrow N\left(0, \sigma^{2}\right) \text { under } P^{\omega} \text { when } n \rightarrow \infty .
$$

where $\bar{S}_{n, n}=S_{n, n}-R_{n, n}$ with $R_{n, n}=E_{n, 0}\left(S_{n, n}\right)+E_{0, n}\left(S_{n, n}\right)-E_{0,0}\left(S_{n, n}\right)$ and

$$
\sigma^{2}=\left\|\sum_{u, v \geq 0} \mathcal{P}_{0,0}\left(X_{u, v}\right)\right\|_{2}^{2}=\lim _{n \wedge m \rightarrow \infty} \frac{E\left(\bar{S}_{n, n}^{2}\right)}{n^{2}} .
$$


In Theorem 2.3 the random centering $R_{n, n}$ cannot be avoided. As a matter of fact, for $d=1$, Volný and Woodroofe (2010) constructed an example showing that the CLT for partial sums need not be quenched. It should also be noticed that, for a stationary ortho-martingale, the existence of finite second moment is not enough for the validity of a quenched CLT when the summation in taken on rectangles (see Peligrad and Volný (2018)). In order to assure the validity of a martingale approximation with a suitable moment condition we shall reinforce condition (77) when dealing with indexes $n$ and $m$ which converge independently to infinity.

Theorem 2.4 Assume now that (7) is reinforced to

$$
\sum_{u, v \geq 0}\left\|\mathcal{P}_{0,0}\left(X_{u, v}\right)\right\|_{\phi}<\infty
$$

where $\phi(x)=x^{2} \log (1+|x|)$ and $\|\cdot\|_{\phi}$ is defined by (4). Then, for almost all $\omega \in \Omega$,

$$
\frac{1}{(n m)^{1 / 2}} \bar{S}_{n, m} \Rightarrow N\left(0, \sigma^{2}\right) \text { under } P^{\omega} \text { when } n \wedge m \rightarrow \infty,
$$

where $\bar{S}_{n, m}=S_{n, m}-R_{n, m}$ with $R_{n, m}=E_{n, 0}\left(S_{n, m}\right)+E_{0, m}\left(S_{n, m}\right)-E_{0,0}\left(S_{n, m}\right)$ and

$$
\sigma^{2}=\left\|\sum_{u, v \geq 0} \mathcal{P}_{0,0}\left(X_{u, v}\right)\right\|_{2}^{2}=\lim _{n \wedge m \rightarrow \infty} \frac{E\left(\bar{S}_{n, m}^{2}\right)}{n m} .
$$

The random centering is not needed if we impose two regularity conditions.

Corollary 2.5 Assume that the conditions of Theorem 2.4 hold. If

$$
\frac{E_{0,0}\left(E_{0, m}^{2}\left(S_{n, m}\right)\right)}{n m} \rightarrow 0 \text { a.s. and } \frac{E_{0,0}\left(E_{n, 0}^{2}\left(S_{n, m}\right)\right)}{n m} \rightarrow 0 \text { a.s. when } n \wedge m \rightarrow \infty \text {, }
$$

then for almost all $\omega \in \Omega$,

$$
\frac{1}{(n m)^{1 / 2}} S_{n, m} \Rightarrow N\left(0, \sigma^{2}\right) \text { under } P^{\omega} \text { when } n \wedge m \rightarrow \infty \text {. }
$$

If the conditions of Theorem 2.3 hold and (10) holds with $m=n$, then for almost all $\omega \in \Omega$,

$$
\frac{1}{n} S_{n, n} \Rightarrow N\left(0, \sigma^{2}\right) \text { under } P^{\omega} \text { when } n \rightarrow \infty .
$$

For the sake of applications, we provide a sufficient condition which takes care of the regularity assumptions (10).

Theorem 2.6 Assume that

$$
\sum_{u, v \geq 1} \frac{\left\|E_{1,1}\left(X_{u, v}\right)\right\|_{2}}{(u v)^{1 / 2}}<\infty .
$$

(a) Then for almost all $\omega \in \Omega$ (12) holds.

(b) If in addition (8) is satisfied, then for almost all $\omega \in \Omega$ (11) holds.

(c) If for some $q>2$

$$
\sum_{u, v \geq 1} \frac{\left\|E_{1,1}\left(X_{u, v}\right)\right\|_{q}}{(u v)^{1 / q}}<\infty
$$

then the quenched convergence in (11) holds.

Remark 2.7 In Corollary 2.5 and Theorem 2.6, $\sigma^{2}$ can be identified as $\sigma^{2}=\lim _{n \wedge m \rightarrow \infty} E S_{n, m}^{2} / n m$ $\left(\lim _{n \rightarrow \infty} E S_{n, n}^{2} / n^{2}\right.$ respectively).

Remark 2.8 Theorem [2.6 can be viewed as an extension to the random fields of Proposition 12 in Cuny and Peligrad (2012). As we shall see, the proof for random fields is much more involved and requires several intermediary steps and new ideas. 


\section{Proofs}

Let us point out the main idea of the proof. Since Peligrad and Volný (2018) proved a quenched CLT for ortho-martingales, we reduce the proof to the existence of an almost sure ortho-martingale approximation. We prove first Theorem 2.4, since the proof of Theorem 2.3 is similar with the exception that we use different ergodic theorems.

Let us denote by $\hat{T}$ and $\hat{S}$ the operators on $L^{2}$ defined by $\hat{T} f=f \circ T, \hat{S} f=f \circ S$.

Proof of Theorem 2.4. Starting from condition (8), by triangle inequality we have that

$$
f_{\mathbf{0}}:=\sum_{u, v \geq 0}\left|\mathcal{P}_{0,0}\left(X_{u, v}\right)\right|<\infty \text { a.s. }
$$

and

$$
\left\|f_{\mathbf{0}}\right\|_{\phi} \leq \sum_{u, v \geq 0}\left\|\mathcal{P}_{0,0}\left(X_{u, v}\right)\right\|_{\phi}<\infty
$$

Note that by (15) $\mathcal{P}_{1,1}\left(S_{n, m}\right)$ is convergent almost surely. Denote the pointwise limit by

$$
D_{1,1}=\lim _{n \wedge m \rightarrow \infty} \mathcal{P}_{1,1}\left(S_{n, m}\right)=\sum_{u, v \geq 1} \mathcal{P}_{1,1}\left(X_{u, v}\right) .
$$

Meanwhile, by the triangle inequality and (8), we obtain

$$
\sup _{n, m \geq 1}\left|\mathcal{P}_{1,1}\left(S_{n, m}\right)\right| \leq \sum_{u, v \geq 1}\left|\mathcal{P}_{1,1}\left(X_{u, v}\right)\right| \text { a.s. }
$$

and

$$
E\left(\sum_{u, v \geq 1}\left|\mathcal{P}_{1,1}\left(X_{u, v}\right)\right|\right)^{2} \leq\left(\sum_{u, v \geq 1}\left\|\mathcal{P}_{1,1}\left(X_{u, v}\right)\right\|_{2}\right)^{2}<\infty .
$$

Thus, by the dominated convergence theorem, $\mathcal{P}_{1,1}\left(S_{n, m}\right)$ converges to $D_{1,1}$ a.s. and in $L^{2}(P)$ as $n \wedge m \rightarrow \infty$.

Since $E_{0,1}\left(\mathcal{P}_{1,1}\left(S_{n, m}\right)\right)=0$ a.s. and $E_{1,0}\left(\mathcal{P}_{1,1}\left(S_{n, m}\right)\right)=0$ a.s., by defining for every $i, j \in Z$, $D_{i, j}=\hat{T}^{i-1} \hat{S}^{j-1} D_{1,1}$, we conclude that $\left(D_{i, j}\right)_{i, j \in Z}$ is a field of martingale differences. By the expression of $D_{1,1}$ above,

$$
D_{i, j}=\sum_{(u, v) \geq(i, j)} \mathcal{P}_{i, j}\left(X_{u, v}\right) .
$$

Now we look into the decomposition of $S_{n, m}$ (see Peligrad and Zhang (2018b) for details):

$$
S_{n, m}-R_{n, m}=\sum_{i=1}^{n} \sum_{j=1}^{m} \mathcal{P}_{i, j}\left(\sum_{u=i}^{n} \sum_{v=j}^{m} X_{u, v}\right)
$$

where

$$
R_{n, m}=E_{n, 0}\left(S_{n, m}\right)+E_{0, m}\left(S_{n, m}\right)-E_{0,0}\left(S_{n, m}\right) .
$$

Therefore

$$
\frac{S_{n, m}-R_{n, m}-M_{n, m}}{\sqrt{n m}}=\frac{1}{\sqrt{n m}} \sum_{i=1}^{n} \sum_{j=1}^{m}\left(\mathcal{P}_{i, j}\left(\sum_{u=i}^{n} \sum_{v=j}^{m} X_{u, v}\right)-D_{i, j}\right) .
$$

By the orthogonality of the field of martingale differences $\left(\mathcal{P}_{i, j}\left(\sum_{u=i}^{n} \sum_{v=j}^{m} X_{u, v}\right)-D_{i, j}\right)_{i, j \in Z}$ and the assumption that the filtration is commuting, we have 


$$
\frac{1}{n m} E_{0,0}\left(S_{n, m}-R_{n, m}-M_{n, m}\right)^{2}=\frac{1}{n m} \sum_{i=1}^{n} \sum_{j=1}^{m} E_{0,0}\left(\mathcal{P}_{i, j}\left(\sum_{u=i}^{n} \sum_{v=j}^{m} X_{u, v}\right)-D_{i, j}\right)^{2} .
$$

From Theorem 1 in Peligrad and Volný (2018), we know that the quenched CLT holds for $M_{n, m} / \sqrt{n m}$. Therefore by Theorem 25.4 in Billingsley (1995), in order to prove the conclusion of this theorem, it is enough to show that

$$
\lim _{n \wedge m \rightarrow \infty} \frac{1}{n m} E_{0,0}\left(S_{n, m}-R_{n, m}-M_{n, m}\right)^{2}=0 \text { a.s. }
$$

Define the operators

$$
Q_{1}(f)=E_{0, \infty}(\hat{T} f) ; Q_{2}(f)=E_{\infty, 0}(\hat{S} f)
$$

Note that $Q_{1}$ and $Q_{2}$ are commuting Dunford-Schwartz operators and we can write

$$
E_{0,0}\left(\mathcal{P}_{i, j}\left(X_{u, v}\right)\right)^{2}=Q_{1}^{i} Q_{2}^{j}\left(\mathcal{P}_{0,0}\left(X_{u-i, v-j}\right)\right)^{2} .
$$

By simple algebra we obtain

$$
\begin{aligned}
& E_{0,0}\left(\mathcal{P}_{i, j}\left(\sum_{u=i}^{n} \sum_{v=j}^{m} X_{u, v}\right)-D_{i, j}\right)^{2} \\
& =E_{0,0}\left(\sum_{u=n+1}^{\infty} \sum_{v=j}^{m} \mathcal{P}_{i, j}\left(X_{u, v}\right)+\sum_{u=i}^{\infty} \sum_{v=m+1}^{\infty} \mathcal{P}_{i, j}\left(X_{u, v}\right)\right)^{2} .
\end{aligned}
$$

Therefore, by elementary inequalities we have the following bound

$$
\begin{aligned}
\frac{1}{n m} E_{0,0}\left(S_{n, m}-R_{n, m}-M_{n, m}\right)^{2} & =\frac{1}{n m} \sum_{i=1}^{n} \sum_{j=1}^{m} E_{0,0}\left(\mathcal{P}_{i, j}\left(\sum_{u=i}^{n} \sum_{v=j}^{m} X_{u, v}\right)-D_{i, j}\right)^{2} \\
& \leq 2\left(I_{n, m}+I I_{n, m}\right),
\end{aligned}
$$

where we have used the notations

$$
I_{n, m}=\frac{1}{n m} \sum_{i=1}^{n} \sum_{j=1}^{m} Q_{1}^{i} Q_{2}^{j}\left(\sum_{u=n+1-i}^{\infty} \sum_{v=0}^{\infty}\left|\mathcal{P}_{0,0}\left(X_{u, v}\right)\right|\right)^{2}
$$

and

$$
I I_{n, m}=\frac{1}{n m} \sum_{i=1}^{n} \sum_{j=1}^{m} Q_{1}^{i} Q_{2}^{j}\left(\sum_{u=0}^{\infty} \sum_{v=m+1-j}^{\infty}\left|\mathcal{P}_{0,0}\left(X_{u, v}\right)\right|\right)^{2} .
$$

The task is now to show the almost sure negligibility of each term. By symmetry we treat only one of them.

Let $c$ be a fixed integer satisfying $c<n$. We decompose $I_{n, m}$ into two parts

$$
\frac{1}{n m} \sum_{i=1}^{n-c} \sum_{j=1}^{m} Q_{1}^{i} Q_{2}^{j}\left(\sum_{u=n+1-i}^{\infty} \sum_{v=0}^{\infty}\left|\mathcal{P}_{0,0}\left(X_{u, v}\right)\right|\right)^{2}:=A_{n, m}(c)
$$

and

$$
\frac{1}{n m} \sum_{i=n-c+1}^{n} \sum_{j=1}^{m} Q_{1}^{i} Q_{2}^{j}\left(\sum_{u=n+1-i}^{\infty} \sum_{v=0}^{\infty}\left|\mathcal{P}_{0,0}\left(X_{u, v}\right)\right|\right)^{2}:=B_{n, m}(c) .
$$


Note that

$$
\begin{aligned}
B_{n, m}(c) & \leq \frac{1}{n m} \sum_{i=n-c+1}^{n} \sum_{j=1}^{m} Q_{1}^{i} Q_{2}^{j} f_{\mathbf{0}}^{2} \\
& =\frac{1}{n m} \sum_{i=1}^{n} \sum_{j=1}^{m} Q_{1}^{i} Q_{2}^{j} f_{\mathbf{0}}^{2}-\frac{1}{n m} \sum_{i=1}^{n-c} \sum_{j=1}^{m} Q_{1}^{i} Q_{2}^{j} f_{\mathbf{0}}^{2},
\end{aligned}
$$

where $f_{0}$ is given by (15).

Since $Q_{1}$ and $Q_{2}$ are commuting Dunford-Schwartz operators, and by (16) we have that $E\left(f_{0}^{2} \log (1+\right.$ $\left.\left.\left|f_{\mathbf{0}}\right|\right)\right)<\infty$, by the ergodic theorem (Krengel (1985), Theorem 1.1, Ch. 6), for each $c$ fixed,

$$
\lim _{n \wedge m \rightarrow \infty} \frac{1}{n m} \sum_{i=1}^{n-c} \sum_{j=1}^{m} Q_{1}^{i} Q_{2}^{j} f_{\mathbf{0}}^{2}=g \text { a.s. }
$$

where

$$
g=\lim _{n \rightarrow \infty} \frac{1}{n} \sum_{i=1}^{n-c} Q_{1}^{i}\left(\lim _{m \rightarrow \infty} \frac{1}{m} \sum_{j=1}^{m} Q_{2}^{j}\left(f_{\mathbf{0}}\right)\right) .
$$

Since we assume that either $S$ or $T$ is ergodic, without loss of generality, here we assume that $S$ is ergodic. By applying Lemma 7.1 in Dedecker et al. (2014), we obtain that

$$
\lim _{m \rightarrow \infty} \frac{1}{m} \sum_{j=1}^{m} Q_{2}^{j}\left(f_{\mathbf{0}}\right)=E\left(f_{\mathbf{0}}^{2}\right) \text { a.s. , }
$$

which implies that $g$ in (21) is a constant almost surely and $g=E\left(f_{\mathbf{0}}^{2}\right)$.

Therefore, for all $c>0$

$$
\lim _{n \wedge m \rightarrow \infty} B_{n, m}(c)=0 \text { a.s. }
$$

In order to treat the first term in the decomposition of $I_{n, m}$, note that

$$
A_{n, m}(c) \leq \frac{1}{n m} \sum_{i=1}^{n-c} \sum_{j=1}^{m} Q_{1}^{i} Q_{2}^{j} f_{\mathbf{0}}^{2}(c) \text { where } f_{\mathbf{0}}(c)=\sum_{u=c}^{\infty} \sum_{v=0}^{\infty}\left|\mathcal{P}_{0,0}\left(X_{u, v}\right)\right|
$$

Again, by the ergodic theorem for Dunford-Schwartz operators (Krengel (1985), Theorem 1.1, Ch. 6) and Lemma 7.1 in Dedecker et al. (2014), for each $c$ fixed

$$
\lim _{n \wedge m \rightarrow \infty} \frac{1}{n m} \sum_{i=1}^{n-c} \sum_{j=1}^{m} Q_{1}^{i} Q_{2}^{j} f_{\mathbf{0}}^{2}(c)=E\left(f_{\mathbf{0}}^{2}(c)\right) \text { a.s. }
$$

In addition, by (15), we know that $\lim _{c \rightarrow \infty}\left|f_{\mathbf{0}}(c)\right|=0$. So, by the dominated convergence theorem, we have

$$
\lim _{c \rightarrow \infty} \lim _{n \wedge m \rightarrow \infty} A_{n, m}(c) \leq \lim _{c \rightarrow \infty} E\left(f_{\mathbf{0}}^{2}(c)\right)=0 \text { a.s. }
$$

The proof of the theorem is now complete.

The proof of Theorem 2.3 requires only a slight modification of the proof of Theorem 2.4. Indeed, instead of Theorem 1.1 in Ch. 6 in Krengel (1985), we shall use Theorem 2.8 in Ch. 6 in the same book.

Proof of Corollary 2.5. By Theorem 2.4 together with Theorem 25.4 in Billingsley (1995), it suffices to show that (10) implies that 


$$
\lim _{n \wedge m \rightarrow \infty} \frac{1}{n m} E_{0,0}\left(R_{n, m}^{2}\right)=0 \text { a.s. }
$$

Simple computations, involving the fact that the filtration is commuting, gives that

$$
E_{0,0}\left(R_{n, m}^{2}\right)=E_{0,0}\left(E_{n, 0}^{2}\left(S_{n, m}\right)\right)+E_{0,0}\left(E_{0, m}^{2}\left(S_{n, m}\right)\right)-E_{0,0}^{2}\left(S_{n, m}\right)
$$

and since $E_{0,0}^{2}\left(S_{n, m}\right) \leq E_{0,0}\left(E_{0, m}^{2}\left(S_{n, m}\right)\right)$ a.s., we have

$$
\lim _{n \wedge m \rightarrow \infty} \frac{1}{n m} E_{0,0}\left(R_{n, m}^{2}\right)=0 \text { a.s. by condition (10). }
$$

We give next the proof of Theorem[2.6. Before proving this theorems, we shall first establish several preliminary facts presented as three lemmas.

Lemma 3.1 Let $q \geq 2$. Condition (14) implies

$$
\sum_{u \geq 1} \frac{1}{u^{1 / q}} \sum_{v \geq 0}\left\|P_{0, \tilde{0}}\left(X_{u, v}\right)\right\|_{q}<\infty .
$$

Proof. Throughout the proof, denote by $C_{q}>0$ a generic constant depending on $q$ which may take different values from line to line. By the Hölder inequality and the Rosenthal inequality for martingales (see Theorem 7.1 in the Appendix), we have

$$
\begin{aligned}
& \sum_{v \geq 1}\left\|P_{0, \tilde{0}}\left(X_{u, v}\right)\right\|_{q}=\sum_{v \geq 1}\left\|P_{-u,-\tilde{v}}\left(X_{0,0}\right)\right\|_{q} \leq \sum_{n \geq 0}\left(2^{n}\right)^{\frac{q-1}{q}}\left(\sum_{v=2^{n}}^{2^{n+1}-1}\left\|P_{-u,-\tilde{v}}\left(X_{0,0}\right)\right\|_{q}^{q}\right)^{\frac{1}{q}} \\
& \quad \leq C_{q} \sum_{n \geq 0}\left(2^{n}\right)^{\frac{q-1}{q}}\left\|\sum_{v=2^{n}}^{2^{n+1}-1} P_{-u,-\tilde{v}}\left(X_{0,0}\right)\right\|_{q} \leq 2 C_{q} \sum_{n \geq 0}\left(2^{n}\right)^{\frac{q-1}{q}}\left\|E_{-u,-2^{n}}\left(X_{0,0}\right)\right\|_{q} .
\end{aligned}
$$

Since the sequence $\left(\left\|E_{-u,-n}\left(X_{0,0}\right)\right\|_{q}\right)_{n \geq 1}$ is non-increasing in $n$, it follows that

$$
\left(2^{n}\right)^{\frac{q-1}{q}}\left\|E_{-u,-2^{n}}\left(X_{0,0}\right)\right\|_{q} \leq 2 \sum_{k=2^{n-1}}^{2^{n}-1} \frac{\left\|E_{-u,-k}\left(X_{0,0}\right)\right\|_{q}}{k^{1 / q}} .
$$

So

$$
\sum_{v=1}^{\infty}\left\|P_{0, \tilde{0}}\left(X_{u, v}\right)\right\|_{q} \leq C_{q} \sum_{k \geq 1} \frac{\left\|E_{-u,-k}\left(X_{0,0}\right)\right\|_{q}}{k^{1 / q}} .
$$

Thus relation (25) holds by (14), (26) and stationarity.

In addition, for any $u \geq 0$, we also have

$$
\sum_{v=1}^{\infty}\left\|P_{0, \tilde{0}}\left(X_{u, v}\right)\right\|_{q}<\infty .
$$

By the symmetric roles of $m$ and $n$, for any $v \geq 0$, we have

$$
\sum_{u=1}^{\infty}\left\|P_{\tilde{0}, 0}\left(X_{u, v}\right)\right\|_{q}<\infty .
$$


Lemma 3.2 Condition (13) implies

$$
\lim _{n \wedge m \rightarrow \infty} \frac{1}{n m} E_{0,0}\left(R_{n, m}^{2}\right)=0 \text { a.s. }
$$

Proof. First we show that (13) implies that

$$
\frac{E_{0,0}^{2}\left(S_{n, m}\right)}{n m} \rightarrow 0 \text { a.s. when } n \wedge m \rightarrow \infty .
$$

We bound this term in the following way

$$
\begin{gathered}
\frac{\left|E_{0,0}\left(S_{n, m}\right)\right|}{\sqrt{n m}} \leq \frac{1}{\sqrt{n m}} \sum_{u=1}^{n} \sum_{v=1}^{m}\left|E_{0,0}\left(X_{u, v}\right)\right| \\
\leq \frac{1}{\sqrt{n}} \sum_{u=1}^{c} \sum_{v=1}^{\infty} \frac{\left|E_{0,0}\left(X_{u, v}\right)\right|}{\sqrt{v}}+\sum_{u=c+1}^{\infty} \sum_{v=1}^{\infty} \frac{\left|E_{0,0}\left(X_{u, v}\right)\right|}{\sqrt{u v}} \\
\leq \frac{c}{\sqrt{n}} \sup _{1 \leq u \leq c} \sum_{v=1}^{\infty} \frac{\left|E_{0,0}\left(X_{u, v}\right)\right|}{\sqrt{v}}+\sum_{u=c+1}^{\infty} \sum_{v=1}^{\infty} \frac{\left|E_{0,0}\left(X_{u, v}\right)\right|}{\sqrt{u v}} .
\end{gathered}
$$

Now, (13) implies that

$$
\sum_{u=1}^{\infty} \sum_{v=1}^{\infty} \frac{\left|E_{0,0}\left(X_{u, v}\right)\right|}{\sqrt{u v}}<\infty \text { a.s. }
$$

Therefore,

$$
\frac{\left|E_{0,0}\left(S_{n, m}\right)\right|}{\sqrt{n m}} \rightarrow 0 \text { a.s. }
$$

by letting $n \rightarrow \infty$ followed by $c \rightarrow \infty$.

By (24) and the symmetric roles of $m$ and $n$, the theorem will follow if we can show that

$$
E_{0,0} \frac{\left(E_{0, m}^{2}\left(S_{n, m}\right)\right)}{n m} \rightarrow 0 \text { a.s. when } n \wedge m \rightarrow \infty .
$$

By (30) this is equivalent to showing that

$$
\frac{1}{n m} E_{0,0}\left(E_{0, m}\left(S_{n, m}\right)-E_{0,0}\left(S_{n, m}\right)\right)^{2} \rightarrow 0 \text { a.s. when } n \wedge m \rightarrow \infty \text {. }
$$

We start from the representation

$$
\begin{aligned}
E_{0,0}\left(E_{0, m}\left(S_{n, m}\right)-E_{0,0}\left(S_{n, m}\right)\right)^{2} & =\sum_{j=1}^{m} E_{0,0}\left[P_{0, \tilde{j}}\left(\sum_{u=1}^{n} \sum_{v=j}^{m} X_{u, v}\right)\right]^{2} \\
& =\sum_{j=1}^{m} E_{0,0}\left[\widehat{S}^{j}\left(P_{0, \tilde{0}}\left(\sum_{u=1}^{n} \sum_{v=0}^{m-j} X_{u, v}\right)\right)^{2}\right] .
\end{aligned}
$$


So,

$$
\begin{gathered}
\frac{1}{n m} E_{0,0}\left(E_{0, m}\left(S_{n, m}\right)-E_{0,0}\left(S_{n, m}\right)\right)^{2}=\frac{1}{m n} \sum_{j=1}^{m} E_{0,0}\left[\widehat{S}^{j}\left(\sum_{u=1}^{n} \sum_{v=0}^{m-j} P_{0, \tilde{0}}\left(X_{u, v}\right)\right)^{2}\right] \\
\leq \frac{2}{m n} \sum_{j=1}^{m} E_{0,0}\left[\widehat{S}^{j}\left(\sum_{u=1}^{c} \sum_{v=0}^{m-j}\left|P_{0, \tilde{0}}\left(X_{u, v}\right)\right|\right)^{2}\right] \\
+\frac{2}{m} \sum_{j=1}^{m} E_{0,0}\left[\widehat{S}^{j}\left(\sum_{u=c+1}^{n} \frac{1}{\sqrt{u}} \sum_{v=0}^{m-j}\left|P_{0, \tilde{0}}\left(X_{u, v}\right)\right|\right)^{2}\right] \\
=I_{n, m, c}+I I_{n, m, c} .
\end{gathered}
$$

Let us introduce the operator

$$
Q_{0}(f)=E_{0,0}(\widehat{S} f) .
$$

We treat first the term $I_{n, m, c}$. For $c$ fixed

$$
\begin{aligned}
I_{n, m, c} & \leq \frac{2 c^{2}}{m n} \sup _{1 \leq u \leq c} \sum_{j=1}^{m} E_{0,0}\left[\widehat{S}^{j}\left(\sum_{v=0}^{\infty}\left|P_{0, \tilde{0}}\left(X_{u, v}\right)\right|\right)^{2}\right] \\
& =\frac{2 c^{2}}{m n} \sup _{1 \leq u \leq c} \sum_{j=1}^{m} Q_{0}^{j}\left[\left(\sum_{v=0}^{\infty}\left|P_{0, \tilde{0}}\left(X_{u, v}\right)\right|\right)^{2}\right] .
\end{aligned}
$$

By (27), the function

$$
g(u)=\sum_{v=0}^{\infty}\left|P_{0, \tilde{0}}\left(X_{u, v}\right)\right|
$$

is square integrable. By the ergodic theorem for Dunford-Schwartz operators (see Theorem 11.4 in Eisner et al., 2015 or Corollary 3.8 in Ch. 3, Krengel, 1985) and Lemma 7.1 in Dedecker et al. (2014),

$$
\frac{1}{m} \sum_{j=1}^{m} Q_{0}^{j}\left[g^{2}(u)\right] \rightarrow E\left(g^{2}(u)\right) \text { a.s. }
$$

and therefore, since $c$ is fixed,

$$
\lim _{n \wedge m \rightarrow \infty} I_{n, m, c}=0 \text { a.s. }
$$

In order to treat the second term, note that

$$
I I_{n, m, c} \leq \frac{2}{m} \sum_{j=1}^{m} Q_{0}^{j}\left[\left(\sum_{u=c}^{\infty} \frac{1}{\sqrt{u}} \sum_{v=0}^{\infty}\left|P_{0, \tilde{0}}\left(X_{u, v}\right)\right|\right)^{2}\right] .
$$

Denote

$$
h(c)=\sum_{u=c}^{\infty} \frac{1}{\sqrt{u}} \sum_{v=0}^{\infty}\left|P_{0, \tilde{0}}\left(X_{u, v}\right)\right| .
$$

By (25), we know that

$$
\sum_{u=1}^{\infty} \frac{1}{\sqrt{u}} \sum_{v=0}^{\infty}\left\|P_{0, \tilde{0}}\left(X_{u, v}\right)\right\|_{2}<\infty .
$$

So, $E\left(h^{2}(c)\right)<\infty$. Again, by the ergodic theorem for the Dunford-Schwartz operators (see Theorem 11.4 in Eisner et al., 2015 or Corollary 3.8 in Ch. 3, Krengel, 1985), we obtain

$$
\frac{1}{m} \sum_{j=1}^{m} Q_{0}^{j}\left(h^{2}(c)\right) \rightarrow E\left(h^{2}(c)\right) \leq\left(\sum_{u=c}^{\infty} \frac{1}{\sqrt{u}} \sum_{v=0}^{\infty}\left\|P_{0, \tilde{1}}\left(X_{u, v}\right)\right\|_{2}\right)^{2} .
$$


So, by (31)

$$
\lim _{c \rightarrow \infty} \lim _{m \rightarrow \infty} \frac{1}{m} \sum_{j=1}^{m} Q_{0}^{j}\left(h^{2}(c)\right)=0 \text { a.s. }
$$

Lemma 3.3 Let $q \geq 2$. Condition [14] implies

$$
\sum_{u, v \geq 0}\left\|\mathcal{P}_{0,0}\left(X_{u, v}\right)\right\|_{q}<\infty
$$

which clearly implies (8).

Proof. By applying twice the Rosenthal inequality for martingales (see Theorem 7.1 in the Appendix), for any integers $a \leq b$ and $c \leq d$, we have

$$
\sum_{k=a}^{b} \sum_{k^{\prime}=c}^{d}\left\|\mathcal{P}_{-k,-k^{\prime}}\left(X_{0,0}\right)\right\|_{q}^{q} \leq C_{q}\left\|\sum_{k=a}^{b} \sum_{k^{\prime}=c}^{d} \mathcal{P}_{-k,-k^{\prime}}\left(X_{0,0}\right)\right\|_{q}^{q} .
$$

In addition, note that for any integers $a \leq b$ and $c \leq d$, we have

$$
\left\|\sum_{k=a}^{b} \sum_{k^{\prime}=c}^{d} \mathcal{P}_{-k,-k^{\prime}}\left(X_{0,0}\right)\right\|_{q}^{q} \leq 4^{q}\left\|E_{-a,-c}\left(X_{0,0}\right)\right\|_{q}^{q} .
$$

Then by the Hölder's inequality together with (33) and (34), we obtain

$$
\begin{gathered}
\sum_{u, v \geq 1}\left\|\mathcal{P}_{-u,-v}\left(X_{0,0}\right)\right\|_{q} \leq \sum_{n, m \geq 0}\left(2^{n} 2^{m}\right)^{\frac{q-1}{q}}\left(\sum_{k=2^{n}}^{2^{n+1}-1} \sum_{k^{\prime}=2^{m}}^{2^{m+1}-1}\left\|\mathcal{P}_{-k,-k^{\prime}}\left(X_{0,0}\right)\right\|_{q}^{q}\right)^{\frac{1}{q}} \\
\leq 4 C_{q} \sum_{n, m \geq 0}\left(2^{n} 2^{m}\right)^{\frac{q-1}{q}}\left\|E_{-2^{n},-2^{m}}\left(X_{0,0}\right)\right\|_{q} .
\end{gathered}
$$

Since $\left\|E_{-2^{n},-2^{m}}\left(X_{0,0}\right)\right\|$ is non-increasing in $n$ and $m$, it follows that

$$
\left(2^{n} 2^{m}\right)^{\frac{q-1}{q}}\left\|E_{-2^{n},-2^{m}}\left(X_{0,0}\right)\right\|_{q} \leq 4 \sum_{u=2^{n-1}}^{2^{n}-1} \sum_{v=2^{m-1}}^{2^{m}-1} \frac{\left\|E_{-u,-v}\left(X_{0,0}\right)\right\|_{q}}{(u v)^{1 / q}} .
$$

Therefore, by the relations above, we have proved that (14) implies

$$
\sum_{u, v \geq 1}\left\|\mathcal{P}_{-u,-v}\left(X_{0,0}\right)\right\|_{q}<\infty
$$

Similarly we have

$$
\sum_{u=1}^{\infty}\left\|\mathcal{P}_{-u, 0}\left(X_{0,0}\right)\right\|_{q}<\infty \text { and } \sum_{v=1}^{\infty}\left\|\mathcal{P}_{0,-v}\left(X_{0,0}\right)\right\|_{q}<\infty .
$$

Thus by stationarity (32) holds.

Proof of Theorem 2.6. The item (a) of the theorem follows as a combination of Theorem 2.3 with Lemmas 3.2 and 3.3, applied with $q=2$.

To prove item (b) of this theorem we combine Theorem 2.4 with Lemmas 3.2 .

Finaly, the conclusion (c) is a consequence of 2.4 combined with Lemmas 3.2 and 3.3 , applied with $q>2$. 


\section{Random fields with multidimensional index sets}

In this section we extend our results to random fields indexed by $Z^{d}, d>2$. By $\mathbf{u} \leq \mathbf{n}$ we understand $\mathbf{u}=\left(u_{1}, \ldots, u_{d}\right), \mathbf{n}=\left(n_{1}, \ldots, n_{d}\right)$ and $1 \leq u_{1} \leq n_{1}, \ldots, 1 \leq u_{d} \leq n_{d}$. We shall start with a strictly stationary real-valued random field $\xi=\left(\xi_{\mathbf{u}}\right)_{\mathbf{u} \in Z^{d}}$, defined on the canonical probability space $R^{Z^{d}}$ and define the filtrations $\mathcal{F}_{\mathbf{u}}=\sigma\left(\xi_{\mathbf{j}}: \mathbf{j} \leq \mathbf{u}\right)$. We shall assume that the filtration is commuting if $E_{\mathbf{u}} E_{\mathbf{a}}(X)=E_{\mathbf{u} \wedge \mathbf{a}}(X)$, where the minimum is taken coordinate-wise and we used notation $E_{\mathbf{u}}(X)=E\left(X \mid \mathcal{F}_{\mathbf{u}}\right)$. We define

$$
X_{\mathbf{m}}=f\left(\left(\xi_{\mathbf{j}}\right)_{\mathbf{j} \leq \mathbf{m}}\right) \text { and set } S_{\mathbf{k}}=\sum_{\mathbf{u}=\mathbf{1}}^{\mathbf{k}} X_{\mathbf{u}} .
$$

The variable $X_{\mathbf{0}}$ is assumed to be square integrable (in $L^{2}$ ) and with mean 0 . We also define $T_{i}$ the coordinate-wise translations and then

$$
X_{\mathbf{k}}=f\left(T_{1}^{k_{1}} \circ \ldots \circ T_{d}^{k_{d}}\left(\xi_{\mathbf{u}}\right)_{\mathbf{u} \leq \mathbf{0}}\right) .
$$

Let $d$ be a function and define

$$
D_{\mathbf{m}}=d\left(\left(\xi_{\mathbf{j}}\right)_{\mathbf{j} \leq \mathbf{m}}\right) \text { and set } M_{\mathbf{k}}=\sum_{\mathbf{u}=\mathbf{1}}^{\mathbf{k}} D_{\mathbf{u}} .
$$

Assume integrability. We say that $\left(D_{\mathbf{m}}\right)_{\mathbf{m} \in Z^{d}}$ is a field of martingale differences if $E_{\mathbf{a}}\left(D_{\mathbf{m}}\right)=0$ if at least one coordinate of $\mathbf{a}$ is strictly smaller than the corresponding coordinate of $\mathbf{m}$. Now we introduce the $d$-dimensional projection operator. By using the fact that the filtration is commuting, it is convenient to define projections $\mathcal{P}_{\mathbf{u}}$ in the following way

$$
\mathcal{P}_{\mathbf{u}}(X):=P_{\mathbf{u}(1)} \circ P_{\mathbf{u}(2)} \circ \ldots \circ P_{\mathbf{u}(d)}(X)
$$

where

$$
P_{\mathbf{u}(j)}(Y):=E\left(Y \mid \mathcal{F}_{\mathbf{u}}\right)-E\left(Y \mid \mathcal{F}_{\mathbf{u}(j)}\right)
$$

where $\mathbf{u}(j)$ has all the coordinates of $\mathbf{u}$ with the exception of the $j$-th coordinate, which is $u_{j}-1$. For instance when $d=3, P_{\mathbf{u}(2)}(Y)=E\left(Y \mid \mathcal{F}_{u_{1}, u_{2}, u_{3}}\right)-E\left(Y \mid \mathcal{F}_{u_{1}, u_{2}-1, u_{3}}\right)$.

We say that a random field $\left(X_{\mathbf{n}}\right)_{\mathbf{n} \in Z^{d}}$ admits a martingale approximation if there is a field of martingale differences $\left(D_{\mathbf{m}}\right)_{\mathbf{m} \in Z^{d}}$ such that for almost all $\omega \in \Omega$

$$
\frac{1}{|\mathbf{n}|} E^{\omega}\left(S_{\mathbf{n}}-M_{\mathbf{n}}\right)^{2} \rightarrow 0 \text { when } \min _{1 \leq i \leq d} n_{i} \rightarrow \infty
$$

where $|\mathbf{n}|=n_{1} \ldots n_{d}$.

Let $R_{\mathbf{n}}$ be the remainder term of the decomposition of $S_{\mathbf{n}}$ such that

$$
S_{\mathbf{n}}=\sum_{\mathbf{u}=\mathbf{1}}^{\mathbf{n}} \mathcal{P}_{\mathbf{u}}\left(S_{\mathbf{n}}\right)+R_{\mathbf{n}} .
$$

In this context we have:

Theorem 4.1 Assume that $\left(X_{\mathbf{n}}\right)_{\mathbf{n} \in Z^{d}}$ is defined by (35) and there is an integer $i, 1 \leq i \leq d$, such that $T_{i}$ is ergodic and the filtrations are commuting. In addition assume that

$$
\sum_{\mathbf{u} \geq \mathbf{0}}\left\|\mathcal{P}_{\mathbf{0}}\left(X_{\mathbf{u}}\right)\right\|_{2}<\infty
$$

Then, for almost all $\omega \in \Omega$,

$$
\left(S_{n, \cdots, n}-R_{n, \cdots, n}\right) / n^{d / 2} \Rightarrow N\left(0, \sigma^{2}\right) \text { under } P^{\omega} \text { when } n \rightarrow \infty .
$$


Theorem 4.2 Furthermore, assume now condition (39) is reinforced to

$$
\sum_{\mathbf{u} \geq \mathbf{0}}\left\|\mathcal{P}_{\mathbf{0}}\left(X_{\mathbf{u}}\right)\right\|_{\varphi}<\infty
$$

where $\varphi(x)=x^{2} \log ^{d-1}(1+|x|)$ and $\|\cdot\|_{\varphi}$ is defined by (4).

Then, for almost all $\omega \in \Omega$,

$$
\frac{1}{\sqrt{|\mathbf{n}|}}\left(S_{\mathbf{n}}-R_{\mathbf{n}}\right) \Rightarrow N\left(0, \sigma^{2}\right) \text { under } P^{\omega} \text { when } \min _{1 \leq i \leq d} n_{i} \rightarrow \infty \text {. }
$$

Corollary 4.3 Assume that the conditions of Theorem 4.2 hold and for all $j, 1 \leq j \leq d$ we have

$$
\frac{1}{|\mathbf{n}|} E_{\mathbf{0}}\left(E_{\mathbf{n}_{j}}^{2}\left(S_{\mathbf{n}}\right)\right) \rightarrow 0 \text { a.s. when } \min _{1 \leq i \leq d} n_{i} \rightarrow \infty \text {. }
$$

where $\mathbf{n}_{j} \in Z^{d}$ has the $j$-th coordinate 0 and the other coordinates equal to the coordinates of $\mathbf{n}$. Then, for almost all $\omega \in \Omega$,

$$
S_{\mathbf{n}} / \sqrt{|\mathbf{n}|} \Rightarrow N\left(0, \sigma^{2}\right) \text { under } P^{\omega} \text { when } \min _{1 \leq i \leq d} n_{i} \rightarrow \infty .
$$

If the conditions of Theorem 4.1 hold and 41) holds with $\mathbf{n}=(n, n, \cdots, n)$, then for almost all $\omega \in \Omega$,

$$
\frac{1}{n^{d / 2}} S_{n, \cdots, n} \Rightarrow N\left(0, \sigma^{2}\right) \text { under } P^{\omega} \text { when } n \rightarrow \infty .
$$

Theorem 4.4 Assume that $\left(X_{\mathbf{n}}\right)_{n \in Z^{d}}$ is defined by (35) and the filtrations are commuting. Also assume that there is an integer $i, 1 \leq i \leq d$, such that $T_{i}$ is ergodic and in addition for $q>2$,

$$
\sum_{\mathbf{u} \geq \mathbf{1}} \frac{\left\|E_{\mathbf{1}}\left(X_{\mathbf{u}}\right)\right\|_{q}}{|\mathbf{u}|^{1 / q}}<\infty
$$

(a) If $q=2$, then the quenched convergence in (43) holds

(b) If $q>2$, then the quenched convergence in (42) holds.

As for the case of random fields with two indexes, we start with the proof of Theorem 4.2, since the proof of Theorem 4.1 is similar with the exception that we use different ergodic theorems.

Proof of Theorem 4.2. The proof of this theorem is straightforward following the same lines of proofs as for a double-indexed random field. It is easy to see that, by using the commutativity property of the filtration, the martingale approximation argument in the proof of Theorem 2.4 remains unchanged if we replace $Z^{2}$ with $Z^{d}$ for $d \geq 3$. The definition of the approximating martingale is also clear. The only difference in the proof is that for the validation of the limit in (21) and (22) when $\min _{1 \leq i \leq d} n_{i} \rightarrow \infty$, in order to apply the ergodic theorem for Dunford-Schwartz operators, conform to Theorem 1.1 in Ch. 6 in Krengel (1985), we have to assume $E\left[f_{\mathbf{0}}^{2} \log ^{d-1}\left(1+\left|f_{\mathbf{0}}\right|\right)\right]<\infty$, which is implied by (40).

More precisely, let us denote by $\hat{T}_{i}, 1 \leq i \leq d$, the operators defined by $\hat{T}_{i} f=f \circ T_{i}$. Then for $\mathbf{i}=\left(i_{1}, \cdots, i_{d}\right) \in Z^{d}$, we define $Q^{\mathbf{i}}=\prod_{k=1}^{d} Q_{k}^{i_{k}}$ where $\left(Q_{i}\right)_{1 \leq i \leq d}$ are operators associated with coordinate-wise translations $\left(T_{i}\right)_{1 \leq i \leq d}$ defined as follows

$$
Q_{1}(f)=E_{0, \infty, \cdots, \infty}\left(\hat{T}_{1} f\right), Q_{2}(f)=E_{\infty, 0, \infty, \cdots, \infty}\left(\hat{T}_{2} f\right), \cdots, Q_{d}(f)=E_{\infty, \cdots, \infty, 0}\left(\hat{T}_{d} f\right) .
$$


Then, we bound the following quantity

$$
\frac{1}{|\mathbf{n}|} E_{\mathbf{0}}\left[\left|S_{\mathbf{n}}-R_{\mathbf{n}}-M_{\mathbf{n}}\right|^{2}\right]
$$

by the sum of $d$ terms with the first term of them in the form

$$
I_{\mathbf{n}}=\frac{1}{|\mathbf{n}|} \sum_{\mathbf{i}=\mathbf{1}}^{\mathbf{n}} \mathbf{Q}^{\mathbf{i}}\left(\sum_{u=n_{1}+1-i_{1}}^{\infty} \sum_{\mathbf{v} \geq \mathbf{0}} \mid \mathcal{P}_{\mathbf{0}}\left(X_{u, \mathbf{v}}\right)\right)^{2} \text { where } \mathbf{v} \in Z^{d-1} .
$$

By symmetry, we only need to deal with this one. Let $c$ be a fixed integer satisfying $c<n_{1}$, we decompose $I_{\mathbf{n}}$ into two parts:

$$
\frac{1}{|\mathbf{n}|} \sum_{i_{1}=1}^{n_{1}-c} \sum_{\mathbf{i}^{\prime}=\mathbf{1}}^{\mathbf{n}^{\prime}} \mathbf{Q}^{\mathbf{i}}\left(\sum_{u=n_{1}+1-i_{1}}^{\infty} \sum_{\mathbf{v} \geq \mathbf{0}}\left|\mathcal{P}_{\mathbf{0}}\left(X_{u, \mathbf{v}}\right)\right|\right)^{2}:=A_{\mathbf{n}}(c)
$$

and

$$
\frac{1}{|\mathbf{n}|} \sum_{i_{1}=n_{1}-c+1}^{n_{1}} \sum_{\mathbf{i}^{\prime}=\mathbf{1}}^{\mathbf{n}^{\prime}} \mathbf{Q}^{\mathbf{i}}\left(\sum_{u=n_{1}+1-i_{1}}^{\infty} \sum_{\mathbf{v} \geq \mathbf{0}} \mid \mathcal{P}_{\mathbf{0}}\left(X_{u, \mathbf{v}}\right)\right)^{2}:=B_{\mathbf{n}}(c)
$$

with $\mathbf{i}^{\prime}=\left(i_{2}, \cdots, i_{d}\right)$ and $\mathbf{n}^{\prime}=\left(n_{2}, \cdots, n_{d}\right)$. Afterwards, we just proceed by following step by step the proof for negligibility of $A_{n, m}(c)$ and $B_{n, m}$ (see (19) and (20) from the proof of Theorem 2.4). The proof of Theorem 4.1 follows by similar arguments, just replacing Theorem 1.1 in Ch. 6 in Krengel (1985) by Theorem 2.8 in Ch. 6 in the same book.

Proof of Corollary 4.3. The negligibility of the reminder $R_{\mathbf{n}}$ can be shown exactly in the same way as the negligibility of the term $R_{n, m}$ in the proof of Corollary 2.5.

Proof of Theorem 4.4. As in the proof of (46) and (47) in Theorem 2.6, we can show that (44) implies the following facts:

$$
\begin{gathered}
\sum_{\mathbf{u} \geq \mathbf{1}} \frac{1}{\sqrt{|\mathbf{u}|}} \sum_{v \geq 0}\left\|P_{\mathbf{0}(d)}\left(X_{\mathbf{u}, v}\right)\right\|_{q}<\infty, \\
\sum_{\mathbf{v} \geq \mathbf{0}}\left\|P_{\mathbf{0}}\left(X_{u, \mathbf{v}}\right)\right\|_{q}<\infty
\end{gathered}
$$

and

$$
\sum_{u \geq 1} \frac{1}{\sqrt{u}} \sum_{\mathbf{v} \geq \mathbf{0}}\left\|P_{\mathbf{0}}\left(X_{u, \mathbf{v}}\right)\right\|_{q}<\infty
$$

where $\mathbf{0}=(0, \cdots, 0) \in Z^{d}, \mathbf{u}, \mathbf{v} \in Z^{d-1}$ and $P_{\mathbf{0}}=P_{\mathbf{0}(2)} \circ P_{\mathbf{0}(3)} \circ \cdots \circ P_{\mathbf{0}(d)}$ with $P_{\mathbf{0}(j)}$ defined by (37).

To prove the corollary, we need to show that

$$
\frac{1}{|\mathbf{n}|} E_{\mathbf{0}}\left(E_{\mathbf{n}^{(k)}}^{2}\left(S_{\mathbf{n}}\right)\right) \rightarrow 0 \text { a.s. when } \min _{1 \leq i \leq d} n_{i} \rightarrow \infty,
$$

where $\mathbf{n}^{(k)} \in Z^{d}$ has $k$ coordinates equal to the corresponding coordinates of $\mathbf{n}$ and the other $n-k$ coordinates zero for all $0 \leq k \leq d-1$. We will proceed by induction.

First, we have to show that

$$
\frac{E_{\mathbf{0}}^{2}\left(S_{\mathbf{n}}\right)}{|\mathbf{n}|} \rightarrow 0 \text { a.s. and } \frac{1}{|\mathbf{n}|} E_{\mathbf{0}}\left(E_{0, \cdots, 0, n_{d}}^{2}\left(S_{\mathbf{n}}\right)\right) \rightarrow 0 \text { a.s. when } \min _{1 \leq i \leq d} n_{i} \rightarrow \infty
$$


which are easy to establish by similar arguments as in the proof of Theorem 2.6. by using (44) and (45). That is, (48) holds for $k=0$ and $k=1$. Now assume that for $k<d-1$ the result holds. The fact that the result holds for $k=d-1$ follows straightforward by using (46) and (47). The proof of this theorem is complete now.

\section{Functional CLT}

In this section we give the functional CLT form for Theorem 2.6. It should be noted that for $d=1$ the quenched functional CLT in the corresponding setting is due to Cuny and Volný (2013). Their approach is based on an almost sure maximal martingale approximation and involves the introduction of two new parameters. This method cannot be easily applied for random fields since it leads to quite complicated remainder terms in the maximal martingale approximation. Fortunately, their innovative idea of using the maximal operator can be also applied for random fields, as we shall see in the direct proof bellow.

For $(s, t) \in[0,1]^{2}$, we introduce the stochastic process

$$
W_{n, m}(t, s)=\frac{1}{\sqrt{n m}} S_{[n t],[m s]} .
$$

where $[x]$ denotes the integer part of $x$. We shall denote by $(W(t, s))_{(t, s) \in[0,1]^{2}}$ the standard 2dimensional Brownian sheet and we shall investigate the weak convergence in $D\left([0,1]^{2}\right)$ endowed with the uniform topology of $\left(W_{n, m}(t, s)\right)$ to $(W(t, s))$. As usual, the proof of this theorem involves two steps, namely the proof of the convergence of the finite dimensional distributions to the corresponding ones of the standard 2-dimensional Brownian sheet and tightness.

We call the random field $\left(X_{k, \ell}\right)$ defined by (3) regular if

$$
E\left(X_{0,0} \mid \mathcal{F}_{0,-\infty}\right)=0 \text { a.s. and } E\left(X_{0,0} \mid \mathcal{F}_{-\infty, 0}\right)=0 \text { a.s. }
$$

Our first result provides a necessary condition for tightness.

Proposition 5.1 Assume that the random field is regular and in addition, for $q>2$, we have

$$
\sum_{i, j \geq 0}\left\|\mathcal{P}_{-i,-j}\left(X_{0,0}\right)\right\|_{q}<\infty .
$$

Then $W_{n, m}(t, s)$ is tight in $D\left([0,1]^{2}\right)$ endowed with the uniform topology.

Proof of Proposition 5.1. We shall start the proof of this theorem by a preliminary consideration: For $2<p<q$, let us introduce the functions

$$
\begin{aligned}
f_{i, j, p}^{*} & =\sup _{n, v \geq 1} \frac{1}{n v} \sup _{n, v} \sum_{k=1}^{n} \sum_{\ell=1}^{v} E^{\omega}\left(\left|\mathcal{P}_{k-i, \ell-j}\left(X_{k, \ell}\right)\right|^{p}\right) \\
& =\sup _{n, v \geq 1} \frac{1}{n v} \sum_{k=1}^{n} \sum_{\ell=1}^{v} Q_{1}^{k} Q_{2}^{\ell}\left(\left|\mathcal{P}_{-i,-j}\left(X_{0,0}\right)\right|^{p}\right) .
\end{aligned}
$$

Let us mention first that, by Corollary 1.7 in Chapter 6 of Krengel (1985) applied to the function $\left|\mathcal{P}_{-i,-j}\left(X_{0,0}\right)\right|^{p}$, for $\lambda>1$ we have

$$
\lambda^{p} P\left(\left(f_{i, j, p}^{*}\right)^{1 / p}>\lambda\right) \leq C E\left(\left|\mathcal{P}_{-i,-j}(0,0)\right|^{p} \log ^{+}\left|\mathcal{P}_{-i,-j}(0,0)\right|\right) \leq C E\left|\mathcal{P}_{-i,-j}\left(X_{0,0}\right)\right|^{q} .
$$

It follows that $\left(f_{i, j, p}^{*}\right)^{1 / p}$ belongs to the weak space $L^{p \text {,weak }}$ defined by

$$
L^{p, \text { weak }}=\left\{f \text { real-valued measurable function defined on } \Omega: \sup _{\lambda>0} \lambda^{p} P(|f|>\lambda)<\infty\right\} .
$$


This is a Banach space whose norm will be denoted by $\|\cdot\|_{p \text {,weak }}$ and it is equivalent to the pseudonorm $\left(\sup _{\lambda>0} \lambda^{p} P(|f|>\lambda)\right)^{1 / p}$. We have that

$$
\left\|\sum_{i, j \geq 0}\left(f_{i, j, p}^{*}\right)^{1 / p}\right\|_{p, \text { weak }} \leq \sum_{i, j \geq 0}\left\|\left(f_{i, j, p}^{*}\right)^{1 / p}\right\|_{p, \text { weak }} \leq \sum_{i, j \geq 0}\left\|\mathcal{P}_{-i,-j}\left(X_{0,0}\right)\right\|_{q} .
$$

Therefore, if $\sum_{i, j \geq 0}\left\|\mathcal{P}_{-i,-j}\left(X_{0,0}\right)\right\|_{q}<\infty$ then

$$
\sum_{i, j \geq 0}\left(f_{i, j, p}^{*}\right)^{1 / p}<\infty P-\text { a.s. }
$$

For proving tightness we shall verify the moment condition given in relation (3) in Bickel and Wichura (1971). To verify it, denote an increment of the process $W_{n, m}(t, s)$ on the rectangle $A=\left[t_{1}, t_{2}\right) \times\left[s_{1}, s_{2}\right)$ by

$$
\Delta(A)=\frac{1}{\sqrt{n m}}\left|\sum_{k=\left[n t_{1}\right]}^{\left[n t_{2}\right]-1} \sum_{\ell=\left[m s_{1}\right]}^{\left[m s_{2}\right]-1} X_{k, \ell}\right| .
$$

Let us note that by (49) we have the representation

$$
X_{k, \ell}=\sum_{i, j \geq 0} \mathcal{P}_{k-i, \ell-j}\left(X_{k, \ell}\right) \text { a.s. }
$$

Fix $\omega$ where this representation holds for all $k$ and $\ell$ and also $\sum_{i, j \geq 0}\left(f_{i, j, p}^{*}\right)^{1 / p}<\infty$. Therefore we have

$$
\|\Delta(A)\|_{\omega, p} \leq \frac{1}{\sqrt{n m}} \sum_{i, j \geq 0}\left\|\sum_{k=\left[n t_{1}\right]}^{\left[n t_{2}\right]-1} \sum_{\ell=\left[m s_{1}\right]}^{\left[m s_{2}\right]-1} \mathcal{P}_{k-i, \ell-j}\left(X_{k, \ell}\right)\right\|_{\omega, p},
$$

where $\|\cdot\|_{\omega, p}$ denotes the norm in $L^{p}\left(P^{\omega}\right)$. Note that, because we have to compute the $p$-th moments of an ortho-martingale, we can use the Burkholder inequality as given in Theorem 3.1 of Fazekas (2005) and obtain

$$
\|\Delta(A)\|_{\omega, p} \leq \frac{C_{p}}{\sqrt{n m}} \sum_{i, j \geq 0}\left\|\sum_{k=\left[n t_{1}\right]}^{\left[n t_{2}\right]-1} \sum_{\ell=\left[m s_{1}\right]}^{\left[m s_{2}\right]-1} \mathcal{P}_{k-i, \ell-j}^{2}\left(X_{k, \ell}\right)\right\|_{\omega, p / 2}^{1 / 2} .
$$

By applying now twice, consecutively, the Cauchy-Schwartz inequality, we obtain

$$
E^{\omega}\left(\Delta^{p}(A)\right) \leq C_{p}\left[\left(t_{2}-t_{1}\right)\left(s_{2}-s_{1}\right)\right]^{p / 2}\left\{\sum_{i, j \geq 0}\left(f_{i, j, p}^{*}(\omega)\right)^{1 / p}\right\}^{p} .
$$

If $B$ is a neighboring rectangle of $A$, by the Hölder inequality we have

$$
E^{\omega}\left(\Delta^{p / 2}(A) \Delta^{p / 2}(B)\right) \leq K_{p, \omega}(\mu(A) \mu(B))^{p / 4},
$$

where $\mu$ is the Lebesgue measure on $[0,1]^{2}$. Therefore the moment condition in relation (3) in Bickel and Wichura (1971) is verified with $\gamma=p$ and $\beta=p / 2$. Since $\beta>1$ the tightness follows from Theorem 3 in Bickel and Wichura (1971).

Theorem 5.2 Assume that (50) and (13) are satisfied. Then for P-almost all $\omega$, the sequence of processes $\left(W_{n, m}(t, s)\right)_{n, m \geq 1}$ converges in distribution on $D\left([0,1]^{2}\right)$ endowed with uniform topology to $\sigma W(t, s)$, as $n \wedge m \rightarrow \infty$ under $P^{\omega}$. 
Proof of Theorem 5.2. The tightness follows by Proposition 5.1. The proof of the convergence of finite dimensional distributions is based on the following observation. By combining the martingale approximation in (18) with the negligibility of $R_{n, m}$ in (29), for almost all $\omega$ and all rational numbers $0 \leq s, t \leq 1$ we obtain

$$
\lim _{n \wedge m \rightarrow \infty} \frac{\left\|S_{[n t],[m s]}-M_{[n t],[m s]}\right\|_{\omega, 2}}{(n m)^{1 / 2}}=0 .
$$

Whence, by using the Cramèr-Wold device and then the triangle inequality, we deduce that the convergence of the finite dimensional distributions follows from the corresponding result for orthomartingales. But this fact was already proved in Peligrad and Volný (2019). The proof is complete.

Similarly we obtain the following result

Theorem 5.3 Assume that (14) is satisfied with $q>2$. Then the conclusion of Theorem 5.2 holds.

Let us formulate the multi-indexed form of this result:

For $\mathbf{t} \in[0,1]^{d}$, where $d$ is fixed a positive integer, we introduce the stochastic random field

$$
W_{\mathbf{n}}(\mathbf{t})=\frac{1}{\sqrt{|\mathbf{n}|}} S_{\left[n_{1} t_{1}\right] \ldots\left[n_{d} t_{d}\right]}
$$

and denote by $(W(\mathbf{t}))_{\mathbf{t} \in[0,1]^{d}}$ the standard $d$-dimensional Brownian sheet. The following is the d-dimensional version of Theorem 5.3 ,

Theorem 5.4 Under the conditions of Theorem 4.4 with $q>2$, for P-almost all $\omega$, the sequence of processes $\left(W_{\mathbf{n}}(\mathbf{t})\right)_{\mathbf{n} \geq \mathbf{1}}$ converges in distribution to $\sigma W(\mathbf{t})$, as $\min _{1 \leq i \leq d} n_{i} \rightarrow \infty$ under $P^{\omega}$.

\section{$6 \quad$ Examples}

We shall give examples providing new results for linear and Volterra random fields. The interest of considering these applications is for obtaining functional quenched CLT by using more general sequences of constants than in Peligrad and Volný (2019), where a coboundary decomposition was used. Let $d$ be an integer greater than 2 and $q>2$. Throughout this section, as before, we denote by $C_{q}>0$ a generic constant depending on $q$, which may be different from line to line.

Example 6.1 (Linear field) Let $\left(\xi_{\mathbf{n}}\right)_{\mathbf{n} \in Z^{d}}$ be a random field of independent, identically distributed random variables, which are centered and $E\left(\left|\xi_{\mathbf{0}}\right|^{q}\right)<\infty$. For $\mathbf{k} \geq \mathbf{0}$ define

$$
X_{\mathbf{k}}=\sum_{\mathbf{j} \geq \mathbf{0}} a_{\mathbf{j}} \xi_{\mathbf{k}-\mathbf{j}}
$$

Assume that

$$
\sum_{\mathbf{k} \geq \mathbf{1}} \frac{1}{|\mathbf{k}|^{1 / q}}\left(\sum_{\mathbf{j} \geq \mathbf{k}-\mathbf{1}} a_{\mathbf{j}}^{2}\right)^{\frac{1}{2}}<\infty .
$$

Then the quenched functional CLT in Theorem 5.4 holds.

Proof. Since

$$
E_{1}\left(X_{\mathbf{k}}\right)=\sum_{\mathbf{j} \geq \mathbf{k}-\mathbf{1}} a_{\mathbf{j}} \xi_{\mathbf{k}-\mathbf{j}}
$$

by the independence of $\xi_{\mathbf{n}}$ and the Rosenthal inequality (see Theorem 7.1, given in the Appendix), we obtain 


$$
\begin{aligned}
\left\|E_{\mathbf{1}}\left(X_{\mathbf{k}}\right)\right\|_{q}^{q} & =\left\|\sum_{\mathbf{j} \geq \mathbf{k}-\mathbf{1}} a_{\mathbf{j}} \xi_{\mathbf{k}-\mathbf{j}}\right\|_{q}^{q} \\
& \leq C_{q}\left[\left(\sum_{\mathbf{j} \geq \mathbf{k}-\mathbf{1}} a_{\mathbf{j}}^{2} E\left(\xi_{\mathbf{k}-\mathbf{j}}^{2}\right)\right)^{\frac{q}{2}}+\sum_{\mathbf{j} \geq \mathbf{k}-\mathbf{1}} E\left|a_{\mathbf{j}} \xi_{\mathbf{k}-\mathbf{j}}\right|^{q}\right] \\
& \leq C_{q}\left[\left(\sum_{\mathbf{j} \geq \mathbf{k}-\mathbf{1}} a_{\mathbf{j}}^{2}\right)^{\frac{q}{2}}\left(E \xi_{\mathbf{0}}^{2}\right)^{\frac{q}{2}}+\sum_{\mathbf{j} \geq \mathbf{k}-\mathbf{1}}\left|a_{\mathbf{j}}\right|^{q} E\left(\left|\xi_{\mathbf{0}}\right|^{q}\right)\right] .
\end{aligned}
$$

By the monotonicity of norms in $\ell_{p}$, we have

$$
\left(\sum_{\mathbf{j} \geq \mathbf{k}-\mathbf{1}}\left|a_{\mathbf{j}}\right|^{q}\right)^{\frac{1}{q}} \leq\left(\sum_{\mathbf{j} \geq \mathbf{k}-\mathbf{1}} a_{\mathbf{j}}^{2}\right)^{\frac{1}{2}} .
$$

Therefore

$$
\left\|E_{\mathbf{1}}\left(X_{\mathbf{k}}\right)\right\|_{q} \leq C_{q}\left(\sum_{\mathbf{j} \geq \mathbf{k}-\mathbf{1}} a_{\mathbf{j}}^{2}\right)^{\frac{1}{2}} .
$$

So condition (44) is implied by (51). Whence the result in Theorem 5.4 holds.

Remark 6.2 For the case $d=2$, we can assume the following condition imposed to the coefficients:

$$
\sum_{\mathbf{k} \geq \mathbf{1}} \frac{1}{|\mathbf{k}|^{1 / 2}}\left(\sum_{\mathbf{j} \geq \mathbf{k}-\mathbf{1}} a_{\mathbf{j}}^{2}\right)^{\frac{1}{2}}<\infty .
$$

(a) If we assume $E\left(\xi_{0}^{2}\right)<\infty$, then the quenched convergence in (12) holds.

(b) If we assume $E\left(\left|\xi_{0}\right|^{2} \log \left(1+\left|\xi_{\mathbf{0}}\right|\right)\right)<\infty$, the quenched convergence in (11) holds.

(c) If we assume $E\left|\xi_{0}\right|^{q}<\infty$, for $q>2$ then, the quench functional CLT in Theorem 5.2, holds.

Proof. The first part of the remark is a direct consequence of item (a) in Theorem [2.6, since condition (13) is implied by (52). To prove (b), notice that by algebraic manipulations similar to those used in the proof of Lemma 3.3, we have that (52) implies that $\sum_{\mathbf{j} \geq \mathbf{0}}\left|a_{\mathbf{j}}\right|<\infty$.

Note that

$$
\mathcal{P}_{0}\left(X_{\mathbf{u}}\right)=a_{\mathbf{u}} \xi_{0}
$$

and therefore we have

$$
\sum_{\mathbf{j} \geq \mathbf{0}}\left\|\mathcal{P}_{\mathbf{0}}\left(X_{\mathbf{j}}\right)\right\|_{\phi}=\sum_{\mathbf{j} \geq \mathbf{0}}\left|a_{\mathbf{j}}\right| \cdot\left\|\xi_{\mathbf{0}}\right\|_{\phi}<\infty .
$$

It follows that (52) also implies (8). Therefore the second part of the remark follows by item (b) in Theorem 2.6. Part (c) of the remark follows in a similar way.

For example, note that (52) holds if we take

$$
a_{u, v}=\frac{1}{u v} \frac{1}{h(u) g(v)},
$$

with $h, g$ slowly varying functions at infinity satisfying

$$
\sum_{u \geq 1} \frac{1}{u h(u)}<\infty \text { and } \sum_{v \geq 1} \frac{1}{v g(v)}<\infty .
$$

Also, we mention that the quenched convergence in (a) does not hold if only $\sum_{\mathbf{j} \geq \mathbf{0}}\left|a_{\mathbf{j}}\right|<\infty$ as shown for $d=1$ in Volný and Woodroofe (2010). 
Example 6.3 (Volterra field) Let $\left(\xi_{\mathbf{n}}\right)_{\mathbf{n} \in Z^{d}}$ be a random field of independent, identically distributed, and centered random variables satisfying $E\left(\left|\xi_{\mathbf{0}}\right|^{q}\right)<\infty$. For $\mathbf{k} \geq \mathbf{0}$, define

$$
X_{\mathbf{k}}=\sum_{(\mathbf{u}, \mathbf{v}) \geq(\mathbf{0}, \mathbf{0})} a_{\mathbf{u}, \mathbf{v}} \xi_{\mathbf{k}-\mathbf{u}} \xi_{\mathbf{k}-\mathbf{v}}
$$

where $a_{\mathbf{u}, \mathbf{v}}$ are real coefficients with $a_{\mathbf{u}, \mathbf{u}}=0$ and $\sum_{\mathbf{u}, \mathbf{v} \geq \mathbf{0}} a_{\mathbf{u}, \mathbf{v}}^{2}<\infty$. In addition, assume that

$$
\sum_{\mathbf{k} \geq \mathbf{1}} \frac{1}{|\mathbf{k}|^{1 / q}}\left(\sum_{\substack{(\mathbf{u}, \mathbf{v}) \geq(\mathbf{k}-\mathbf{1}, \mathbf{k}-\mathbf{1}) \\ \mathbf{u} \neq \mathbf{v}}} a_{\mathbf{u}, \mathbf{v}}^{2}\right)^{1 / 2}<\infty .
$$

Then the quenched functional CLT in Theorem 5.4 holds.

Proof. Note that

$$
E_{\mathbf{1}}\left(X_{\mathbf{k}}\right)=\sum_{(\mathbf{u}, \mathbf{v}) \geq(\mathbf{k}-\mathbf{1}, \mathbf{k}-\mathbf{1})} a_{\mathbf{u}, \mathbf{v}} \xi_{\mathbf{k}-\mathbf{u}} \xi_{\mathbf{k}-\mathbf{v}}
$$

Let $\left(\xi_{\mathbf{n}}^{\prime}\right)_{\mathbf{n} \in Z^{d}}$ and $\left(\xi_{\mathbf{n}}^{\prime \prime}\right)_{\mathbf{n} \in Z^{d}}$ be two independent copies of $\left(\xi_{\mathbf{n}}\right)_{\mathbf{n} \in Z^{d}}$. By independence and the fact that $a_{\mathbf{k}, \mathbf{k}}=0$, by applying the decoupling inequality together with the Rosenthal inequality, both of which are given for convenience in the Appendix, (see Theorem 7.2 and Theorem 7.1 from the Appendix), we obtain

$$
\begin{aligned}
& \left\|E_{\mathbf{1}}\left(X_{\mathbf{k}}\right)\right\|_{q}^{q}=\left\|\sum_{\substack{(\mathbf{u}, \mathbf{v}) \geq(\mathbf{k}-1, \mathbf{k}-\mathbf{1}) \\
\mathbf{u} \neq \mathbf{v}}} a_{\mathbf{u}, \mathbf{v}} \xi_{\mathbf{k}-\mathbf{u}} \xi_{\mathbf{k}-\mathbf{v}}\right\|_{q}^{q} \leq C_{2}\left\|\sum_{\substack{(\mathbf{u}, \mathbf{v}) \geq(\mathbf{k}-\mathbf{1}, \mathbf{k}-\mathbf{1}) \\
\mathbf{u} \neq \mathbf{v}}} a_{\mathbf{u}, \mathbf{v}} \xi_{\mathbf{k}-\mathbf{u}}^{\prime} \xi_{\mathbf{k}-\mathbf{v}}^{\prime \prime}\right\|_{q}^{q} \\
& \leq C_{q}\left[\left(\sum_{\substack{(\mathbf{u}, \mathbf{v}) \geq(\mathbf{k}-\mathbf{1}, \mathbf{k}-\mathbf{1}) \\
\mathbf{u} \neq \mathbf{v}}} a_{\mathbf{u}, \mathbf{v}}^{2} E\left(\xi_{\mathbf{k}-\mathbf{u}}^{\prime} \xi_{\mathbf{k}-\mathbf{v}}^{\prime \prime}\right)^{2}\right)^{\frac{q}{2}}+\sum_{\substack{(\mathbf{u}, \mathbf{v}) \geq(\mathbf{k}-\mathbf{1}, \mathbf{k}-\mathbf{1}) \\
\mathbf{u} \neq \mathbf{v}}}\left|a_{\mathbf{u}, \mathbf{v}}\right|^{q} E\left(\left|\xi_{\mathbf{k}-\mathbf{u}}^{\prime} \xi_{\mathbf{k}-\mathbf{v}}^{\prime \prime}\right|^{q}\right)\right]
\end{aligned}
$$

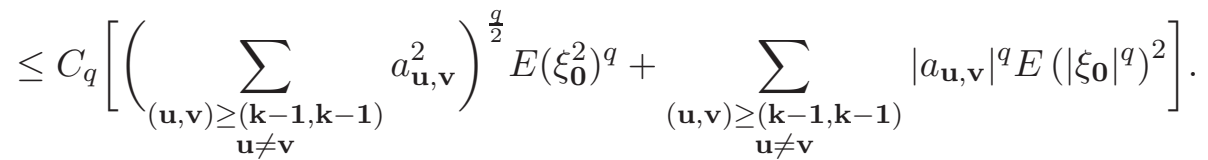

Above, the first inequality holds by Theorem 7.2 while the second one is implied by Theorem 7.1 . Again by the monotonicity of norms in $\ell_{p}$, we have

$$
\left\|E_{\mathbf{1}}\left(X_{\mathbf{k}}\right)\right\|_{q} \leq C_{q}\left(\sum_{\mathbf{u}, \mathbf{v} \geq \mathbf{k}-\mathbf{1}} a_{\mathbf{u}, \mathbf{v}}^{2}\right)^{\frac{1}{2}} .
$$

Thus the results of Theorem 5.4 hold.

\section{Appendix}

For convenience, we mention one classical inequality for martingales, see Theorem 2.11, p. 23, Hall and Heyde (1980) and also Theorem 6.6.7 Ch. 6, p. 322, de la Peña and Giné (1999).

Theorem 7.1 (Rosenthal's Inequality) Let $p \geq 2$. Let $M_{n}=\sum_{k=1}^{n} X_{k}$ where $\left\{M_{n}, \mathcal{F}_{n}\right\}$ is a martingale with martingale differences $\left(X_{n}\right)$. Then there are constants $0<c_{p}, C_{p}<\infty$ such that 


$$
\begin{aligned}
& c_{p}\left\{\sum_{k=1}^{n} E\left|X_{k}\right|^{p}+E\left[\left(\sum_{k=1}^{n} E\left(X_{k}^{2} \mid \mathcal{F}_{k-1}\right)\right)^{p / 2}\right]\right\} \\
& \leq\left\|M_{n}\right\|_{p}^{p} \leq C_{p}\left\{E\left[\left(\sum_{k=1}^{n} E\left(X_{k}^{2} \mid \mathcal{F}_{k-1}\right)\right)^{p / 2}\right]+\sum_{k=1}^{n} E\left|X_{k}\right|^{p}\right\} .
\end{aligned}
$$

The following theorem is a decoupling result for U-statistics, which can be found on p. 99, Theorem 3.1.1, de la Peña and Giné (1999).

Theorem 7.2 (Decoupling inequality) Let $\left(X_{i}\right)_{1 \leq i \leq n}$ be $n$ independent random variables and let $\left(X_{i}^{k}\right)_{1 \leq i \leq n}, k=1, \cdots, m$, be $m$ independent copies of this sequences. For each $\left(i_{1}, i_{2}, \cdots, i_{m}\right) \in$ $I_{n}^{m}$, let $h_{i_{1}, \cdots, i_{m}}: R^{m} \rightarrow R$ be a measurable function such $E\left|h_{i_{1}, \cdots, i_{m}}\left(X_{i_{1}}, \cdots, X_{i_{m}}\right)\right|<\infty$. Let $f:$ $[0, \infty) \rightarrow[0, \infty)$ be a convex non-decreasing function such that $E f\left(\left|h_{i_{1}, \cdots, i_{m}}\left(X_{i_{1}}, \cdots, X_{i_{m}}\right)\right|\right)<\infty$ for all $\left(i_{1}, i_{2}, \cdots, i_{m}\right) \in I_{n}^{m}$, where $I_{n}^{m}=\left\{\left(i_{1}, \cdots, i_{m}\right): i_{j} \in \mathbb{N}, 1 \leq i_{j} \leq n, i_{j} \neq i_{k}\right.$, if $\left.j \neq k\right\}$. Then there exists $C_{m}>0$ such that

$$
\operatorname{Ef}\left(\left|\sum_{I_{n}^{m}} h_{i_{1}, \cdots, i_{m}}\left(X_{i_{1}}, \cdots, X_{i_{m}}\right)\right|\right) \leq \operatorname{Ef}\left(C_{m}\left|\sum_{I_{n}^{m}} h_{i_{1}, \cdots, i_{m}}\left(X_{i_{1}}^{1}, \cdots, X_{i_{m}}^{m}\right)\right|\right) .
$$

\section{Acknowledgements}

This research was supported in part by the NSF grant DMS-1811373. Also we acknowledge a support from the Taft research center at the University of Cincinnati and a grant provided by MIIS (Mathématiques, Information, Ingénierie des Systèmes), Université de Rouen Normandie. The authors are grateful to Dalibor Volný for carefully reading the manuscript and useful discussions, and to the referee for suggestions that contributed to an improvement of a previous version of the paper.

\section{References}

[1] Barrera, D., Peligrad, C.and Peligrad, M. (2016). On the functional CLT for stationary Markov chains started at a point. Stochastic Process. Appl. 126, 1885-1900.

[2] Bickel, P.J. and Wichura, M.J. (1971). Convergence criteria for multiparameter stochastic processes and some applications. Ann. Math. Statist. 42, 1656-1670.

[3] Billingsley, P. (1995). Probability and Measures. (3rd ed.). Wiley Series in Probability and Statistics, New York.

[4] Cairoli, R. (1969). Un théorème de convergence pour martingales a indices multiples. C. R. Acad. Sci. Paris Sér. A-B 269, A587-A589.

[5] Cuny, C. and Peligrad, M. (2012). Central limit theorem started at a point for stationary processes and additive functional of reversible J. Theoret. Probab. 25, 171-188.

[6] Cuny, C. and Merlevède, F. (2014). On martingale approximations and the quenched weak invariance principle. Ann. Probab. 42, 760-793.

[7] Cuny, C., Dedecker J. and Volný, D. (2015). A functional central limit theorem for fields of commuting transformations via martingale approximation. J. Math. Sci. 219, 765-781. 
[8] Cuny, C. and Volný, D. (2013). A quenched invariance principle for stationary processes. ALEA Lat. Am. J. Probab. Math. Stat. 10, 107-115.

[9] De la Peña, V. and Giné, E. (1999). Decoupling : from dependence to independence. Randomly stopped processes. U-statistics and processes. Martingales and beyond. Springer series in statistics. Probability and its applications. Springer, New York.

[10] Dedecker, J., Merlevède, F. and Peligrad, M. (2014). A quenched weak invariance principle. Annales de l'I.H.P. Probabilités et statistiques 50, 872-898.

[11] Derriennic, Y. and Lin, M. (2001). The central limit thorem for Markov chains with normal transition operators started at a point. Probab. Theory Relat. Fields 119, 508-528.

[12] Eisner, T., Farkas, B., Haase, M. and Nagel, R. (2015). Operator theoretic aspects of ergodic theory. Graduate Texts in Mathematics. Springer, Cham.

[13] El Machkouri, M., Volný, D. and Wu, W.B. (2013). A central limit theorem for stationary random fields. Stochastic Process. Appl. 123, 1-14.

[14] Fazekas, I. (2005). Burkholder's inequality for multiindex martingales, Ann. Math. Inform. 32, $45-51$.

[15] Giraudo, D. (2017). Invariance principle via ortho-martingale approximation. Stochastics and Dynamics. Online Ready.

[16] Gordin, M. I. (2009). Martingale-coboundary representation for a class of stationary random fields. Zap. Nauchn. Sem. S.-Peterburg. Otdel. Mat. Inst. Steklov. (POMI) 364, Veroyatnostn i Statistika. 14.2, 88-108, 236; and J. Math. Sci. (N. Y.) 163, 363-374.

[17] Hall, P. and Heyde, C. C. (1980). Martingale Limit Theory and Its Application. Probability and Mathematical Statistics. Academic Press, Inc. [Harcourt Brace Jovanovich, Publishers], New York-London.

[18] Hannan, E. J. (1973). Central limit theorems for time series regression. Z. Wahrscheinlichkeitstheorie und Verw. Gebiete 26, 157-170.

[19] Krasnosel'skii, M.A. and Rutitskii, Ya.B. (1961). Convex functions and Orlicz spaces (Translated from the first Russian edition). P. Noordhoff Ltd., Groningen.

[20] Krengel, U. (1985). Ergodic Theorems. De Gruyter Studies in Mathematics, Berlin.

[21] Peligrad, M. (2015). Quenched Invariance Principles via Martingale Approximation; in Asymptotic laws and methods in stochastics. The volume in honour of Miklos Csorgo work. Springer in the Fields Institute Communications Series. Springer-Verlag New York 76, 121-137.

[22] Peligrad, M. and Zhang, Na (2018a). On the normal approximation for random fields via martingale methods. Stoch. Proc. Appl. 128, 1333-1346.

[23] Peligrad, M. and Zhang, Na (2018b). Martingale approximation for random fields. Electron. Commun. Probab. 23, Page NO. 28, 9.

[24] Peligrad, M. and Volný, D. (2018). Quenched invariance principles for ortho-martingale-like sequences. arXiv:1802.09106 [math.PR]. J. Theor. Probab. (online first).

[25] Rosenblatt, M. (1972). Central limit theorem for stationary processes. Proc. Sixth Berkeley Symp. on Math. Statist. and Prob. Vol. 2 (Univ. of Calif. Press), 551-561. 
[26] Volný, D. (2015). A central limit theorem for fields of martingale differences, C. R. Math. Acad. Sci. Paris 353, 1159-1163.

[27] Volný, D. and Wang, Y. (2014). An invariance principle for stationary random fields under Hannan's condition. Stochastic Proc. Appl. 124, 4012-4029.

[28] Volný, D. and Woodroofe, M. (2010). An example of non-quenched convergence in the conditional central limit theorem for partial sums of a linear process. Dependence in analysis, probability and number theory (The Phillipp memorial volume), Kendrick Press. 317-323.

[29] Volný, D. and Woodroofe, M. (2014). Quenched central limit theorems for sums of stationary processes. Statist. Probab. Lett. 85, 161-167. 